\title{
All-optical time-resolved measurement of laser energy modulation in a relativistic electron beam
}

\author{
D. Xiang, E. Colby, M. Dunning, S. Gilevich, C. Hast, K. Jobe, D. McCormick, J. Nelson, T. O. Raubenheimer, \\ K. Soong, G. Stupakov, Z. Szalata, D. Walz, S. Weathersby, and M. Woodley \\ SLAC National Accelerator Laboratory, Menlo Park, California 94025, USA \\ (Received 17 September 2011; published 18 November 2011)
}

\begin{abstract}
We propose and demonstrate an all-optical method to measure laser energy modulation in a relativistic electron beam. In this scheme the time-dependent energy modulation generated from the electron-laser interaction in an undulator is converted into time-dependent density modulation with a chicane, which is measured to infer the laser energy modulation. The method, in principle, is capable of simultaneously providing information on femtosecond time scale and $10^{-5}$ energy scale not accessible with conventional methods. We anticipate that this method may have wide applications in many laser-based advanced beam manipulation techniques.
\end{abstract}

DOI: 10.1103/PhysRevSTAB.14.112801

PACS numbers: 29.27.Fh, 41.60.Cr

\section{INTRODUCTION}

Many advanced beam manipulation techniques require generation of energy modulation in beam phase space with lasers, i.e., the laser slicing method for generation of femtosecond (fs) $\mathrm{x}$-ray pulses in synchrotrons [1,2], staged laser accelerator with phase stable net acceleration $[3,4]$, laser-assisted beam conditioning [5], mode locking for free electron lasers (FELs) [6], optical replica synthesizer for characterizing beam longitudinal phase space [7], FELs with external seeding for generation of fully coherent short-wavelength radiation [8-12], current-enhanced selfamplified spontaneous emission FELs [13], beat-wave narrow-band $\mathrm{THz}$ emission from laser-modulated beams [14], etc. Accurate measurement of the time-dependent laser energy modulation $\Delta E(t)$ is essential to achieve optimal performance of these techniques.

The conventional method uses an rf transverse cavity (TCAV) (for measuring $t$ ) together with an energy spectrometer (to determine $E$ ) to measure the time-dependent energy distribution of a relativistic electron beam [15-17]. In the conventional method, the electron's energy is converted to the transverse position with a dispersive element and is measured with a view screen. Limited by the intrinsic beam size due to the transverse emittance and the dispersion function of the spectrometer, the energy resolution is about $\sqrt{\epsilon_{n} \beta / \gamma} / \eta$, where $\epsilon_{n}$ is the normalized emittance, $\beta$ is the beta function in the bending plane at the screen, $\gamma$ is the relativistic energy, and $\eta$ is the dispersion of the spectrometer. Furthermore, when a TCAV is used to streak the beam in time, the transverse accelerating field in a TCAV will increase the beam relative slice energy spread by

Published by the American Physical Society under the terms of the Creative Commons Attribution 3.0 License. Further distribution of this work must maintain attribution to the author(s) and the published article's title, journal citation, and DOI.

$$
\sigma_{\delta}=\frac{\epsilon_{n}}{\gamma c \Delta t}
$$

where $\Delta t$ is the temporal resolution of the measurement and is defined as

$$
\Delta t=\frac{\sigma^{\prime} \lambda_{r f} E}{2 \pi c e V},
$$

where $\sigma^{\prime}$ is the intrinsic rms beam divergence in the streaking plane, $\lambda_{\mathrm{rf}}$ is the wavelength of the rf field, $V$ is the voltage of the TCAV, $E$ is the beam energy, and $c$ is the speed of light. This growth in energy spread from the TCAV further limits the resolution in measurements of slice energy spread and laser energy modulation, regardless of the dispersion of the spectrometer. Obviously, if a stronger streak is used to achieve a higher temporal resolution, the energy resolution is worse. Henceforth, it is difficult to simultaneously achieve both high temporal resolution and high energy resolution with this conventional method.

The work reported here pursues an all-optical method to measure the time-dependent laser energy modulation. In this method, a dispersive element (i.e. 4-dipole chicane), typically required in most of the laser-based advanced beam manipulation techniques, is used to convert the time-dependent energy modulation into time-dependent density modulation, which is measured with a TCAV to infer the laser energy modulation amplitude. Because with this method a particle's energy is converted into the longitudinal position before the beam passes through the TCAV and the particles' longitudinal positions do not change in the TCAV, it is immune to both the transverse emittance and the transverse acceleration effects in the TCAV, allowing both high temporal resolution and high energy resolution to be achieved simultaneously. The technique has been routinely used to provide accurate information for the energy modulation in a recent echo-enabled harmonic generation experiment [18] performed at SLAC's Next 
Linear Collider Test Accelerator (NLCTA). We anticipate that this method will find applications in many laser-based advanced beam manipulation techniques.

\section{METHODS}

It is well known that, when a laser interacts with a relativistic beam in an undulator, it generates sinusoidal energy modulation in the electron beam. Consider a coasting beam with constant current of which the initial longitudinal phase space distribution is $f_{0}(p)$ $=N_{0}(2 \pi)^{-1 / 2} e^{-p^{2} / 2}$, where $N_{0}$ is the number of electrons per unit length, $p=\left(E-E_{0}\right) / \sigma_{E}$ is the dimensionless energy deviation of a particle, $E_{0}$ is the average beam energy, and $\sigma_{E}$ is the rms energy spread. For simplicity it is further assumed that the laser has infinitely long duration such that, after interacting with the beam in an undulator, the particle's energy changes to $p^{\prime}=p+A \sin (k z)$, where $A=\Delta E / \sigma_{E}, \Delta E$ is the energy modulation amplitude which is also constant, $k$ is the wave number of the laser, and $z$ is the longitudinal coordinate in the beam. The distribution function after the interaction with the laser becomes $f_{1}(z, p)=N_{0}(2 \pi)^{-1 / 2} \exp \left[-(p-A \sin k z)^{2} / 2\right]$.

After passing through a chicane with momentum compaction $R_{56}$, a particle's longitudinal position changes to $z^{\prime}=z+R_{56} p \sigma_{E} / E_{0}$ (where $p$ now refers to the value at the entrance to the chicane), and yields the distribution function

$f_{f}(z, p)=\frac{N_{0}}{\sqrt{2 \pi}} \exp \left[-\frac{1}{2}\left[p-A_{1} \sin \left(k z-p R_{56} k \sigma_{E} / E_{0}\right)\right]^{2}\right]$.

As a result of this transformation, the energy modulation is converted into density modulation. Accordingly, a beam that initially has constant density now consists of many bumps equally separated by the laser wavelength.

Integration of Eq. (3) over $p$ gives the beam density $N$ as a function of $z, N(z)=\int_{-\infty}^{\infty} d p f_{f}(z, p)$. One important parameter that characterizes the density modulation is the bunching factor $b_{n}$, which is defined as $b_{n}=$ $\frac{1}{N_{0}}\left|\left\langle e^{-i n k z} N(z)\right\rangle\right|$, where $n$ is the harmonic number, and the brackets denote averaging over the coordinate $z$. The bunching factor at various harmonic numbers is [8]

$$
b_{n}=\left|J_{n}\left(n k R_{56} \Delta E / E\right)\right| e^{-(1 / 2) n^{2} k^{2} R_{56}^{2} \sigma_{E}^{2} / E^{2}} .
$$

Take the beam at SLAC's NLCTA as an example, the beam energy is $E=120 \mathrm{MeV}$ and the nominal beam slice energy spread is $\sigma_{E}=1 \mathrm{keV}$. Assuming $R_{56}=6 \mathrm{~mm}$ for the chicane and $\lambda=795 \mathrm{~nm}$ for the laser, the bunching at the fundamental frequency $(n=1)$ for various energy modulations is shown in Fig. 1.

Figure 1 shows that the bunching factor oscillates as a function of energy modulation, as dictated by the Bessel function in Eq. (4). The bunching at the fundamental

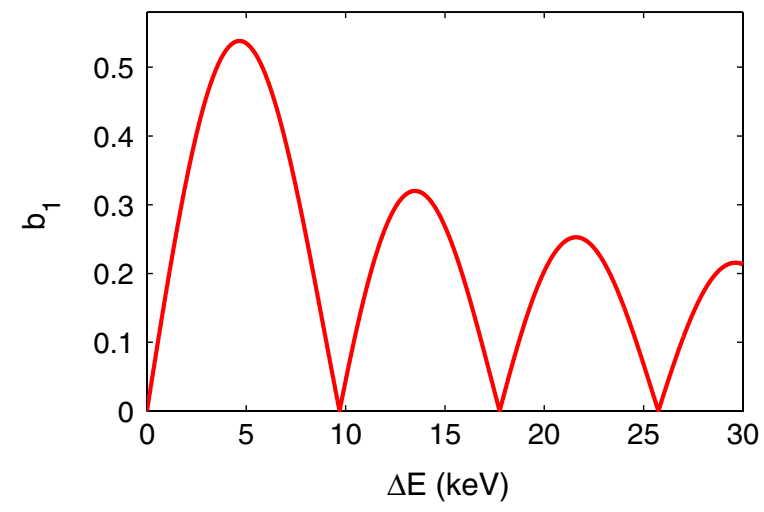

FIG. 1. Bunching at the fundamental frequency for various energy modulations.

frequency peaks approximately when $2 R_{56} \Delta E / E \approx(m+$ $1 / 2) \lambda$ and achieves the minima when $2 R_{56} \Delta E / E \approx m \lambda$, where $m$ is a non-negative integer. The first peak of the bunching at $\Delta E \approx 4.66 \mathrm{keV}$, the second peak at $\Delta E \approx$ $13.49 \mathrm{keV}$, and the third peak at $\Delta E \approx 21.60 \mathrm{keV}$ correspond to the cases when the particles in the peak and those in the valley of the energy modulation initially separated by $\lambda / 2,3 \lambda / 2$, and $5 \lambda / 2$ appear roughly at the same longitudinal position after passing through the chicane, as illustrated in Fig. 2.

In realistic scenarios typically a laser with finite duration is used to modulate the beam. Assuming a Gaussian laser with rms pulse width $\sigma_{t}$, the energy modulation amplitude can be written as $\Delta E(t)=\Delta E \exp \left(-t^{2} / 4 \sigma_{t}^{2}\right)$, where $\Delta E$ is the peak modulation in the center of the laser pulse. In this case, the bunching factor depends on the longitudinal position of the beam and the time-dependent bunching factor $b_{n}(t)$ is given by Eq. (4), with $\Delta E$ replaced by $\Delta E(t)$. For a Gaussian laser with $\sigma_{t}=0.3 \mathrm{ps}$, given the same parameters used in Fig. 1 and a peak energy modulation of $15 \mathrm{keV}$, the bunching at the fundamental frequency for various $R_{56}$ is shown in Fig. 3.

For small $R_{56}$, when $k R_{56} \Delta E / E \ll 1$, we have $b_{1}(t) \propto$ $\Delta E(t)$, and the time-dependent bunching has a similar shape as the laser pulse (magenta line in Fig. 3). With a large $R_{56}$ such that $k R_{56} \Delta E / E \gg 1$, the bunching factor will oscillate along the longitudinal direction (blue line and red line in Fig. 3). More peaks and dips will show up as the $R_{56}$ is increased. Alternatively, given some $R_{56}$, more oscillations will occur if the peak energy modulation is increased.

Since the energy modulations for the peaks and dips of the oscillations are well defined by the Bessel function in Eq. (4) in the regime when energy modulation is much larger than energy spread [the exponential term in Eq. (4) is close to unity], measuring the time-dependent bunching at a specific harmonic may allow one to obtain the timedependent energy modulation generated by a laser in a relativistic electron beam. 

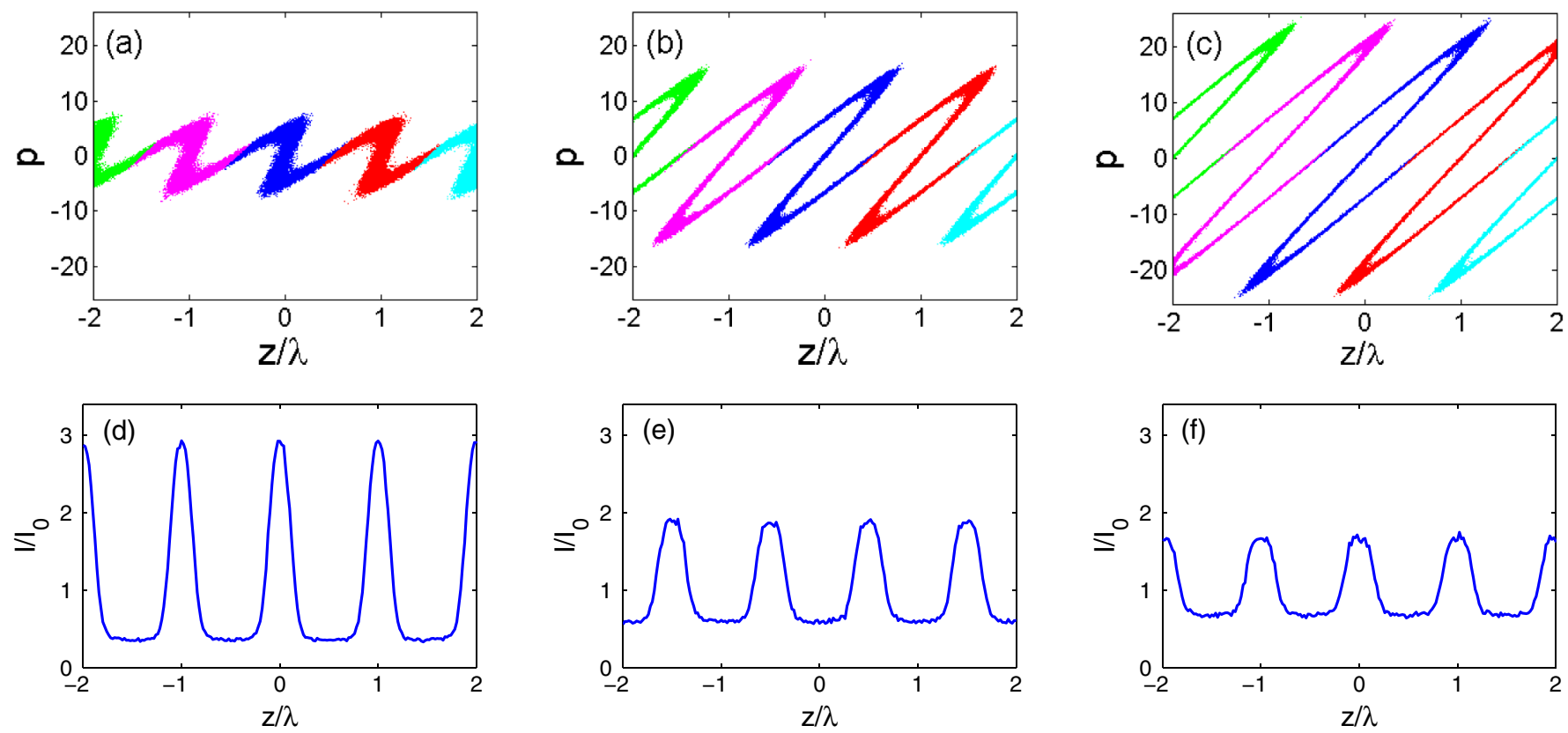

FIG. 2. Longitudinal phase space and current distribution after the chicane when $\Delta E=4.66 \mathrm{keV}[(\mathrm{a})$ and (d) $], \Delta E=13.49 \mathrm{keV}[(\mathrm{b})$ and (e)], and $\Delta E=21.60 \mathrm{keV}$ [(c) and (f)], respectively. Particles initially within the same wavelength are marked with the same color.

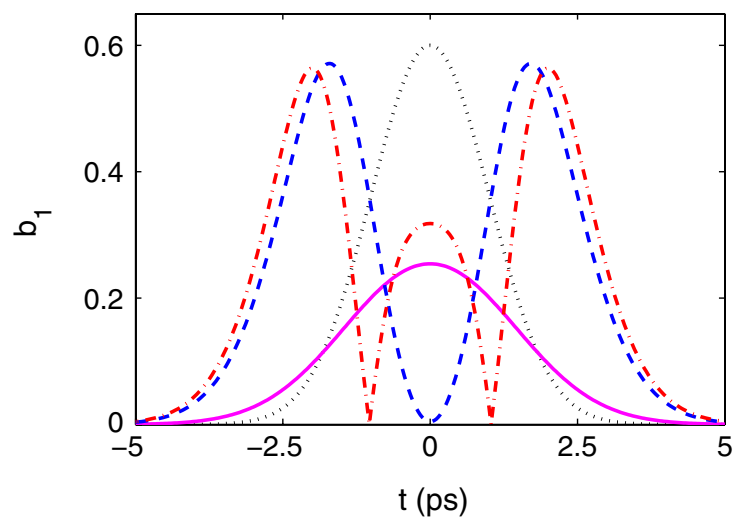

FIG. 3. Time-dependent bunching at the fundamental frequency for various $R_{56}$ : magenta line for $R_{56}=0.4 \mathrm{~mm}$; blue line for $R_{56}=2.9 \mathrm{~mm}$; red line for $R_{56}=3.8 \mathrm{~mm}$; black line for the laser profile.

It is worth mentioning that, while the above analysis focuses on $n=1$, the time-dependent energy modulation can be obtained by measuring the time-dependent bunching for the higher harmonics $(n>1)$ as well. From a practical point of view, it is desirable to choose the suitable $n$ to facilitate the measurement. For instance, in the experiment below, we have obtained the energy modulation from a $1590 \mathrm{~nm}$ laser by measuring the time-dependent bunching at the 2nd harmonic of the laser, where sensitive detectors in the optical wavelengths are available.

\section{EXPERIMENT}

The experiment using the above proposed alloptical method to measure laser energy modulation is schematically shown in Fig. 4. This experiment was conducted at SLAC's NLCTA using the beam line developed for the echo-enabled harmonic generation experiment [12]. The electron beam with about $20 \mathrm{pC}$ charge is generated in a 1.6 cell S-band photocathode rf gun with a frequencytripled $(266 \mathrm{~nm})$ Ti:sapphire laser and is boosted to $120 \mathrm{MeV}$ with two X-band linac sections. In this experiment, a $1590 \mathrm{~nm}$ laser was used to generate energy modulation in the electron beam, and this energy modulation was converted to density modulation after a chicane. The density-modulated beam was first streaked vertically with a 27-cell X-band TCAV, and then passed through a thin aluminum foil to generate coherent optical transition radiation (COTR) at the laser frequency and its harmonics. The COTR intensity at the 2nd harmonic of the laser was measured and further used to infer the energy modulation.

Using the procedures as described in [12], the electron beam was made to overlap with the laser both spatially and temporally in the undulator (10 periods with a period of $5.5 \mathrm{~cm}$ and a $K$ value of 2.09), so that effective beam-laser interaction is achieved to generate energy modulation in

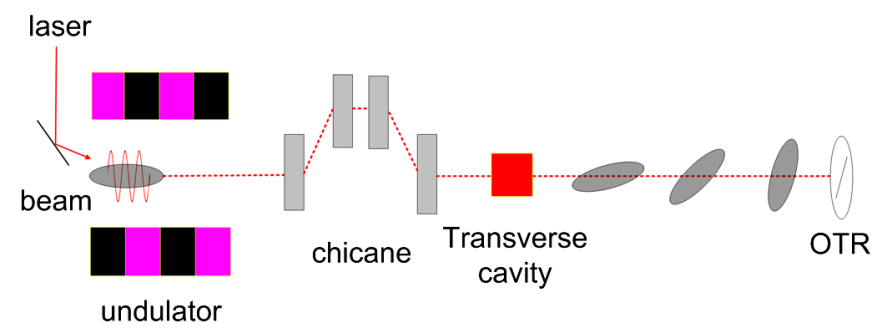

FIG. 4. Schematic of the all-optical time-resolved measurement of laser energy modulation. 
the electron beam. In this experiment, the beam energy spread is less than $1 \%$, well within the bandwidth of the undulator. Therefore, the detuning effect is negligible and the energy modulation is solely determined by the laser intensity.

The TCAV was then used to imprint a time-dependent angular kick $\left(y^{\prime} \propto t\right)$ on the beam. After about 90 degrees phase advance in the vertical plane, the angular distribution is converted to spatial distribution, and the vertical axis on the OTR screen downstream of the cavity becomes the time axis $(y \propto t)$. The absolute time is calibrated by scanning the TCAV phase (e.g., \pm 5.4 degrees) and recording the vertical beam centroid motion on the screen. The calibration coefficient is about $180 \mathrm{fs} / \mathrm{mm}$ on the OTR screen. With the TCAV off, the rms beam size on the OTR screen is about $230 \mu \mathrm{m}$, corresponding to a temporal resolution of about $40 \mathrm{fs}$ in this experiment.

The electron beam temporal profile was first measured with the TCAV and the result is shown in Fig. 5. The head of the bunch (direction of time) was identified by increasing the beam energy such that it has a shorter path length in the chicane and thus enters the TCAV at an earlier time. The timing of the laser is adjusted in such a way that it interacts with the central part of the beam.

To measure the radiation at the 2 nd harmonic of the laser, a bandpass filter centered at $795 \mathrm{~nm}$ with a bandpass of $160 \mathrm{~nm}$ is used in the measurement to block the radiation at other harmonics. The measured time-resolved COTR signal for various $R_{56}$ of the chicane is shown in Fig. 6 .

It can be seen from Fig. 6 that more oscillations were generated when the chicane $R_{56}$ was increased, and up to six oscillations were observed when chicane $R_{56}$ was set at $7.2 \mathrm{~mm}$. Note, the time-dependent COTR intensity in the 1D limit $\left(\gamma \lambda \gg \sigma_{r}\right.$, where $\lambda$ is the radiation wavelength and $\sigma_{r}$ is the transverse beam size) is proportional to $I(t)^{2} b_{2}(t)^{2}$, where $I(t)$ is the beam current distribution. The current distribution has been measured to be a smooth function as shown in Fig. 5; the oscillation of the COTR intensity in Fig. 6 is therefore attributed to the oscillation of the bunching factor. When $3 \mathrm{D}$ effects are taken into

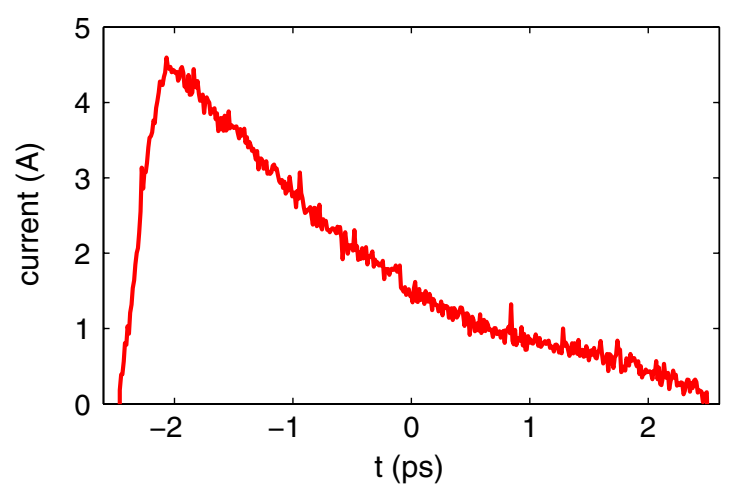

FIG. 5. Beam current distribution (head of the bunch to the left). (a)

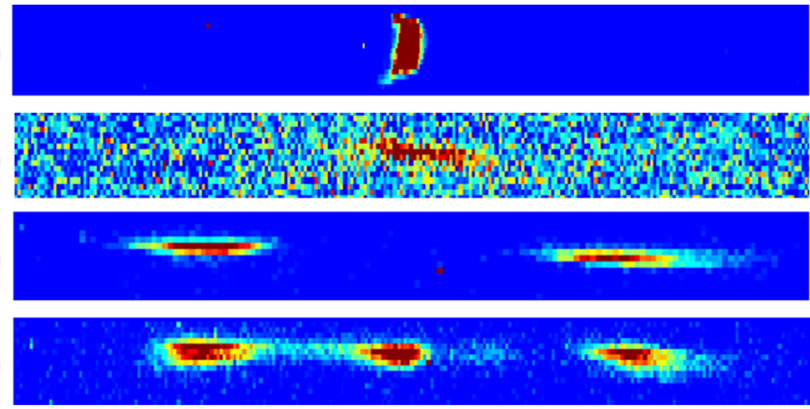

(e)

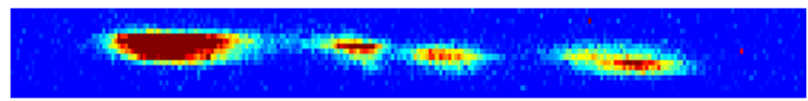

(f)

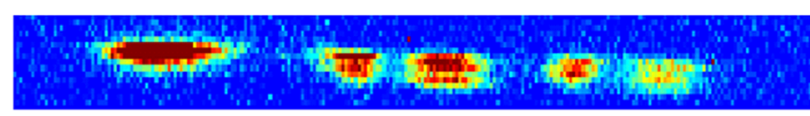

(g)

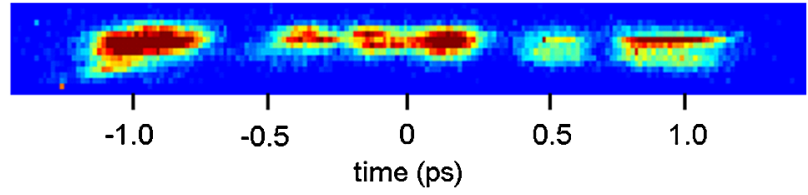

FIG. 6. Time-resolved measurement of the COTR intensity at $795 \mathrm{~nm}$ (head of the bunch to the left): (a) with TCAV off and $R_{56}=7.2 \mathrm{~mm}$; (b) $-(\mathrm{g})$ with $\mathrm{TCAV}$ on and $R_{56}$ set at 1.3, 2.4, 3.5, $4.8,5.6$, and $7.2 \mathrm{~mm}$, respectively.

account, the COTR intensity also depends on beam transverse size [19]. Since in this experiment the laser is much shorter than the beam, it only interacts with a short slice of the beam. Therefore, it is justified to assume that the beam transverse size does not change dramatically in the region of interaction and the differences in COTR intensity are dominated by the variations of the bunching factor. Figure 6 also indicates that the COTR signal is stronger in the head than in the tail. This is correlated with the fact that the beam current is higher in the head.

Given $R_{56}=7.2 \mathrm{~mm}$, the bunching factor at the $2 \mathrm{nd}$ harmonic of the $1590 \mathrm{~nm}$ laser for various energy modulations is shown in Fig. 7. From Eq. (4) it is found that the first three peaks (marked as 1p, 2p, and 3p in Fig. 7) of $b_{2}$ correspond to energy modulations of $6.44,14.14$, and $21.02 \mathrm{keV}$, respectively; and the first two dips (marked as $1 \mathrm{~d}$ and $2 \mathrm{~d}$ in Fig. 7) of $b_{2}$ correspond to energy modulations of $10.83 \mathrm{keV}$ and $17.75 \mathrm{keV}$, respectively.

To retrieve the time-dependent energy modulation, the projected time-resolved COTR intensity in Fig. 6(g) is obtained and shown in Fig. 8. Assuming the timedependent energy modulation has a quasi-Gaussian distribution such that it peaks in the center and decays monotonically in the head and tail, the order of the peaks and dips is identified, as labeled in Fig. 8. The assumption of a quasi-Gaussian distribution for the laser modulation is justified because typically a laser with a bandwidth close to the transform limit is used in the above-mentioned advanced beam manipulation methods. However, it should be pointed out that if the energy modulation is imposed 


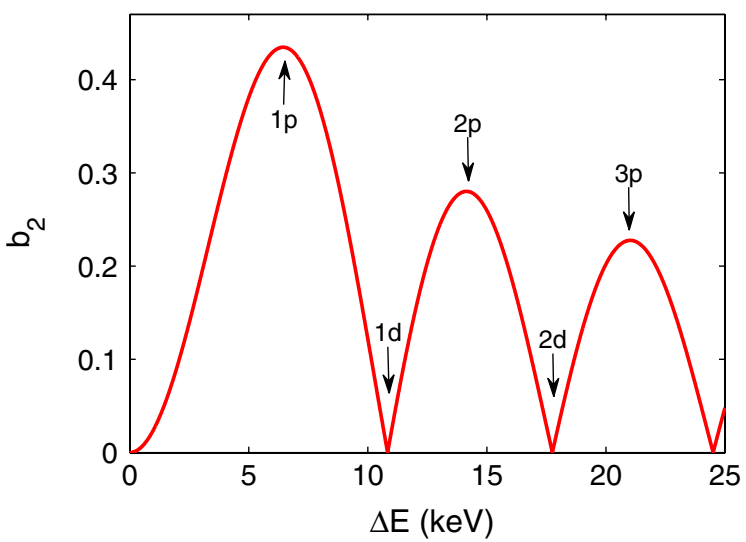

FIG. 7. Bunching at the 2nd harmonic of the $1590 \mathrm{~nm}$ laser for various energy modulations.

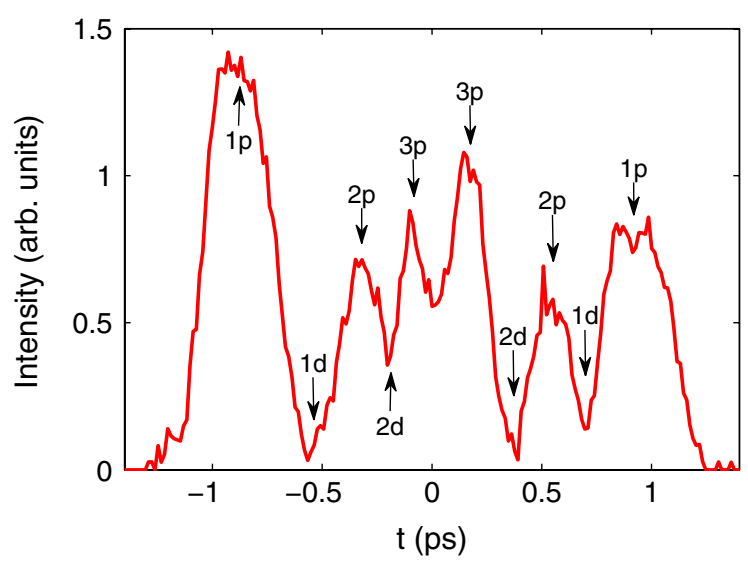

FIG. 8. Projected time-dependent COTR intensity for Fig. 6(g).

with a laser having a complicated shape (i.e. double-horn shape), the assumption does not hold and it may become difficult to accurately retrieve the time-dependent energy modulation.

From Fig. 8 we obtain $\Delta E(t)$ at several discrete values and the retrieved time-resolved energy modulation is shown in Fig. 9(a). Accordingly, the peak energy modulation is quantified to be about $22.5 \mathrm{keV}$. Similarly, the temporal profile of the $1590 \mathrm{~nm}$ laser is reconstructed (assuming the laser power is proportional to the square of the energy modulation) and shown in Fig. 9(b). The FWHM of the laser pulse is found to be about $0.7 \mathrm{ps}$. It should be pointed out that the temporal resolution in the measurement of laser profile with this method is limited by the slippage effect in the undulator (the corresponding resolution is about $50 \mathrm{fs}$ in this experiment).

\section{SUMMARY AND OUTLOOK}

In summary, an all-optical method has been demonstrated which allows time-resolved measurement of laser energy modulation on the electron beam. In this scheme, the time-dependent energy modulation is converted into a time-dependent density modulation which is further measured and used to infer the amplitude of the laser energy modulation. By converting $E$ to $z$, the method becomes immune to both the transverse emittance and the transverse acceleration effects in a TCAV. The method can also be used to measure the temporal profile of a laser pulse. It can be especially useful for determining the pulse shape for those lasers where suitable nonlinear crystals are absent.

The temporal resolution of the technique is limited by the TCAV voltage and beam emittance. In principle, increasing the TCAV voltage and reducing beam emittance should increase the resolution to a few fs. The energy resolution of this method is ultimately limited by beam slice energy spread, because when energy modulation is comparable to or smaller than the beam energy spread, the bunching factor will strongly depend on the slice energy spread and one cannot retrieve the energy modulation from the peaks and dips of the bunching. Taking advantage of the small energy spread of our beam, an energy resolution of a few $\mathrm{keV}$ was achieved in this experiment.

The all-optical method proposed and demonstrated in this paper is expected to be able to simultaneously provide information on femtosecond time scale and $10^{-5}$ energy scale, and we anticipate that this method will find applications in many laser-based advanced beam manipulation techniques.
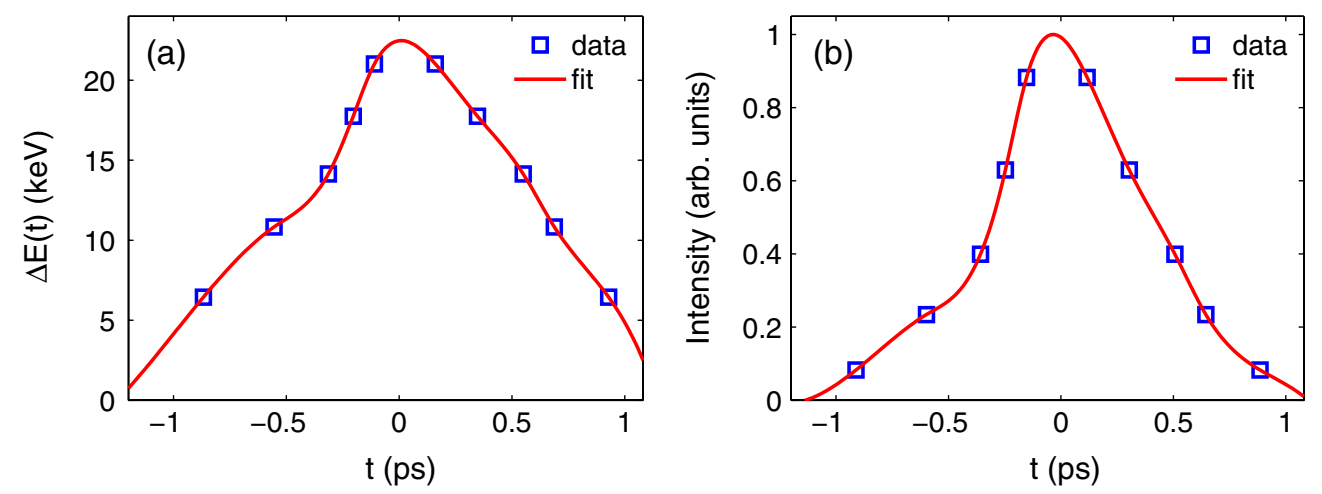

FIG. 9. (a) Reconstructed time-dependent energy modulation; (b) reconstructed temporal profile for the $1590 \mathrm{~nm}$ laser. 


\section{ACKNOWLEDGMENTS}

This work was supported by the U.S. DOE Office of Basic Energy Sciences using the NLCTA facility which is partly supported by U.S. DOE Office of High Energy Physics under Contract No. DE-AC02-76SF00515.

Note added.-After completion of this work, we became aware of a recent observation at the FERMI@ Elettra FEL, where a double spectrum was generated when the seed laser energy was increased [20]. We believe this observation is similar to that in our experiment, and it can be explained with the time-dependent bunching of which the central part of the beam is overbunched and the bunch head and tail are optimally bunched to give the two peaks.

[1] A. Zholents and M. Zolotorev, Phys. Rev. Lett. 76, 912 (1996).

[2] R. Schoenlein, S. Chattopadhyay, H. Chong, T. Glover, P. Heimann, C. Shank, A. Zholents, and M. Zolotorev, Science 287, 2237 (2000).

[3] W. Kimura, A. van Steenbergen, M. Babzien, I. Ben-Zvi, L. Campbell, D. Cline, C. Dilley, J. Gallardo, S. Gottschalk, P. He, K. Kusche, Y. Liu, R. Pantell, I. Pogorelsky, D. Quimby, J. Skaritka, L. Steinhauer, and V. Yakimenko, Phys. Rev. Lett. 86, 4041 (2001).

[4] C. Sears, E. Colby, R. England, R. Ischebeck, C. McGuinness, J. Nelson, R. Noble, R. Siemann, J. Spencer, D. Walz, T. Plettner, and R. Byer, Phys. Rev. ST Accel. Beams 11, 101301 (2008).

[5] A. Zholents, Phys. Rev. ST Accel. Beams 8, 050701 (2005).

[6] N. Thompson and B. McNeil, Phys. Rev. Lett. 100, 203901 (2008).

[7] E. Saldin, E. Schneidmiller, and M. Yurkov, Nucl. Instrum. Methods Phys. Res., Sect. A 539, 499 (2005).

[8] L.-H. Yu, Phys. Rev. A 44, 5178 (1991).
[9] L.-H. Yu, M. Babzien, I. Ben-Zvi, L. DiMauro, A. Doyuran, W. Graves, E. Johnson, S. Krinsky, R. Malone, I. Pogorelsky, J. Skaritka, G. Rakowsky, L. Solomon, X. J. Wang, M. Woodle, V. Yakimenko, S. Biedron, J. N. Galayda, E. Gluskin, J. Jagger, V. Sajaev, and I. Vasserman, Science 289, 932 (2000).

[10] G. Stupakov, Phys. Rev. Lett. 102, 074801 (2009).

[11] D. Xiang and G. Stupakov, Phys. Rev. ST Accel. Beams 12, 030702 (2009).

[12] D. Xiang, E. Colby, M. Dunning, S. Gilevich, C. Hast, K. Jobe, D. McCormick, J. Nelson, T. O. Raubenheimer, K. Soong, G. Stupakov, Z. Szalata, D. Walz, S. Weathersby, M. Woodley, and P.-L. Pernet, Phys. Rev. Lett. 105, 114801 (2010).

[13] A. Zholents, Phys. Rev. ST Accel. Beams 8, 040701 (2005).

[14] D. Xiang and G. Stupakov, Phys. Rev. ST Accel. Beams 12, 080701 (2009).

[15] Z. Huang, A. Brachmann, F.-J. Decker, Y. Ding, D. Dowell, P. Emma, J. Frisch, S. Gilevich, G. Hays, Ph. Hering, R. Iverson, H. Loos, A. Miahnahri, H.-D. Nuhn, D. Ratner, G. Stupakov, J. Turner, J. Welch, W. White, J. Wu, and D. Xiang, Phys. Rev. ST Accel. Beams 13, 020703 (2010).

[16] R. Akre, D. Dowell, P. Emma, J. Frisch, S. Gilevich, G. Hays, Ph. Hering, R. Iverson, C. Limborg-Deprey, H. Loos, A. Miahnahri, J. Schmerge, J. Turner, J. Welch, W. White, and J. Wu, Phys. Rev. ST Accel. Beams 11, 030703 (2008).

[17] M. Röhrs, C. Gerth, H. Schlarb, B. Schmidt, and Peter Schmüser, Phys. Rev. ST Accel. Beams 12, 050704 (2009).

[18] D. Xiang, E. Colby, M. Dunning, S. Gilevich, C. Hast, K. Jobe, D. McCormick, J. Nelson, T. O. Raubenheimer, K. Soong, G. Stupakov, Z. Szalata, D. Walz, S. Weathersby, and M. Woodley (unpublished).

[19] A. Tremaine, J. Rosenzweig, S. Anderson, P. Frigola, M. Hogan, A. Murokh, C. Pellegrini, D. Nguyen, and R. Sheffield, Phys. Rev. Lett. 81, 5816 (1998).

[20] E. Allaria, in Proceedings of FEL11, Shanghai (to be published). 